\title{
Period Variation of the Pop.II Cepheid AU Peg
}

\author{
J. Vinko \\ JATE University, Szeged, and Konkoly Observatory, Budapest, Hungary
}

\begin{abstract}
AU Peg, a short period BL Her-type variable, is unique among short period Cepheids. It is a member of a close binary system with an unseen companion. In $t$ his paper the pulsational period variation is presented. It is shown that the period variation is very rapid and highly non-linear. It seems that there is no simple explanation of this phenomena by standard evolution or tidal interaction.
\end{abstract}

\section{Introduction}

The Population II Cepheid AU Peg is a metal-rich BL Her-type variable and a member of a close binary system which has the shortest orbital period among the known systems containing a Cepheid variable. Its binary nature was discovered from radial velo city measurements and the orbit was computed by Harris et al. $(1979,1984)$. The pulsating component is peculiar and it shows extremely rapid period change (Szabados 1977).

\section{Period Variation}

We have investigated the time dependence of the period variation from available photometric measurements. Constructing the $\mathrm{O}-\mathrm{C}$ diagram, Fourier-spectra and phase dispersion minimization spectra of the individual light curves, seasonal periods were determined. The period variation is non-linear (Fig. 1) and there are at least two points where the rate of the period variation changed almost abruptly. From the most recent data (Szabados \& Vinko 1992) it seems that the period increase stopped and the period began to decrease. It is of interest that this 'break' coincides with the appearance of a slight resonance between the orbital and the pulsational pe$\operatorname{riod}\left(P_{\mathrm{orb}} / P_{\mathrm{pul}} \sim 22\right)$. Such a period variation is unprecedented among short period Cepheids. Since this star is located outside the instability strip (Harris et al. 1984) it has been suspected that the companion has a major effect on the pulsation of AU Peg. The presence of circumstellar matter (McAlary \& Welch 1986) seems to strengthen this hypothesis. However, the period change is so complicated that it can hardly be explained by simple tidal interaction or stellar evolution alone. Continuous 
observations and detailed hydrodynamical modelling are desirable.

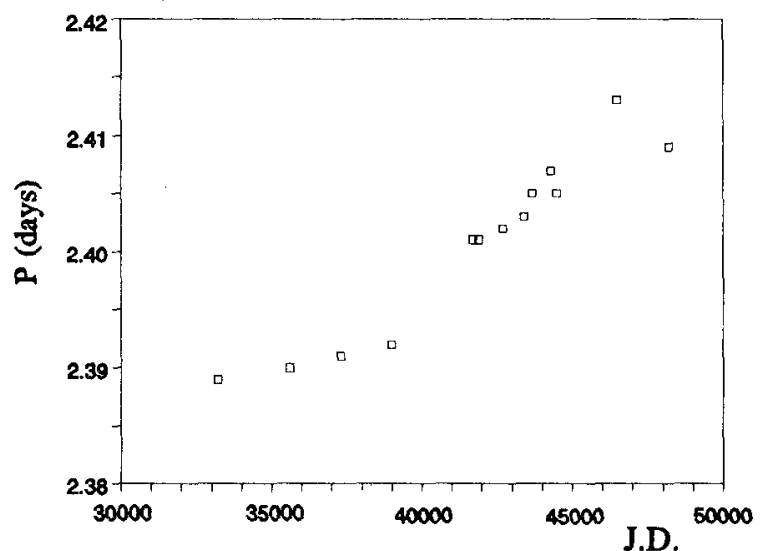

Fig. 1. Pulsational period variation of AU Peg. The error bars are smaller than the symbols.

\section{References:}

Harris, H.C., Olszewski, E. \& Wallerstein, G. 1977, Astron.J. 84, 1598.

Harris, H.C., Olszewski, E. \& Wallerstein, G. 1984, Astron.J. 89, 119.

McAlary, C.W. \& Welch, D.L. 1986, Astron. J. 91, 1209.

Szabados, L. 1977, Mitt. Sternw. Ung. Akad. Wiss. No.79.

Szabados, L. \& Vinko, J. 1992, in preparation

\section{Discussion:}

D.WELCH: It is interesting that the three known binary Pop.II Cepheids are all metal rich. For AU Peg, Harris showed that the period change was consistent with the mass exchange.

J.VINKO: Yes, the mass transfer could cause many complications in the pulsation. T.KREIDL: The Ap star ET And (a $\delta$ Sct pulsator) is in an eccentric, very close binary with even shorter $P_{\text {orb. }}$. During a few years of observation we see no evide nce of period change, so the duplicity may not be influencing any period change in $\mathrm{AU}$ Peg as well.

E.BÖHM-VITENSE: Binary Cepheids with $P_{\text {orb }}<100$ days cannot exist because of the size of the stars. What does $P_{\text {orb }}=50$ days tell you about the evolutionary state of this star?

C.WAELKENS: A Pop.II Cepheid must have been a red giant before, and it could not be in such a close system. Could this star be a Pop.I Cepheid caught during a first crossing?

J.VINKO: AU Peg star is definitely a Pop.II Cepheid because of its galactic position. I agree that AU Peg could not be a red giant in this system. It may be that AU Peg had a unique evol ution due to the mass transfer between the components. But the evolutionary state of the metal-rich BL Her stars are quite uncertain. 